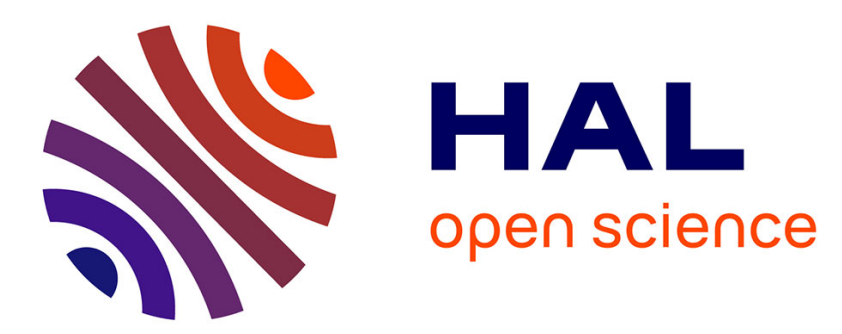

\title{
Vibration of a stiffened pipe filled with a bubbly liquid: analysis of resonance frequencies in function of bubble fraction
}

Sanae Serbout, Laurent Maxit, Frédéric Michel

\section{- To cite this version:}

Sanae Serbout, Laurent Maxit, Frédéric Michel. Vibration of a stiffened pipe filled with a bubbly liquid: analysis of resonance frequencies in function of bubble fraction. Inter-noise 2021, Aug 2021, Washington, France. 10.3397/IN-2021-1730 . hal-03427207

\section{HAL Id: hal-03427207 https://hal.science/hal-03427207}

Submitted on 13 Nov 2021

HAL is a multi-disciplinary open access archive for the deposit and dissemination of scientific research documents, whether they are published or not. The documents may come from teaching and research institutions in France or abroad, or from public or private research centers.
L'archive ouverte pluridisciplinaire HAL, est destinée au dépôt et à la diffusion de documents scientifiques de niveau recherche, publiés ou non, émanant des établissements d'enseignement et de recherche français ou étrangers, des laboratoires publics ou privés. 


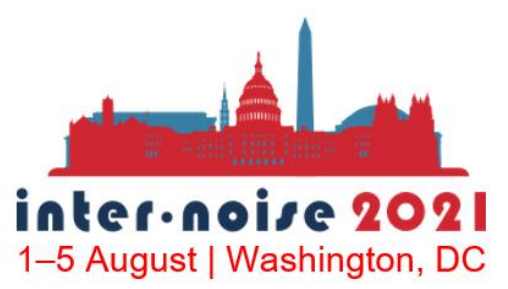

\title{
VIBRATION OF A STIFFENED PIPE FILLED WITH A BUBBLY LIQUID: ANALYSIS OF RESONANCE FREQUENCIES IN FUNC- TION OF BUBBLE FRACTION
}

\author{
Sanae SERBOUT ${ }^{1}$ \\ CEA, DES, IRESNE, Nuclear Technology Department, \\ Cadarache F-13108 Saint-Paul-Lez-Durance, France \\ Laurent MAXIT ${ }^{2}$ \\ Laboratoire Vibrations-Acoustique (LVA), \\ INSA Lyon, Villeurbanne, France \\ Frédéric MICHEL ${ }^{3}$ \\ CEA, DES, IRESNE, Nuclear Technology Department, \\ Cadarache F-13108 Saint-Paul-Lez-Durance, France
}

\begin{abstract}
The characterization of the presence of bubbles in industrial fluid circuits may be extremely important for many safety issues. It is well known that the acoustic properties of liquids can be drastically modified by a small amount of gas content in the liquid. At sufficiently low frequencies, the sound velocity depends primarily on the gas volume fraction. The variation of the gas fraction may then induce some variations in the vibroacoustic behavior of the pipe transporting the liquid. Analysis of the pipe vibrations can then help in the monitoring of the bubble presence. In such a context, the aim of this study is to show how the presence of bubbles in the liquid could affect the resonance frequencies of the pipe. A numerical vibroacoustic model has been developed to predict the vibroacoustic behavior of a stiffened cylindrical shell filled with bubbly liquid exhibiting low frequency resonances. The model is numerically verified for the shell without the liquid by a finite element simulation and then used to analyze the frequency shifts of the shell resonances in function of the void fraction.
\end{abstract}

\footnotetext{
${ }^{1}$ Sanae.serbout@cea.fr

${ }^{2}$ Laurent.maxit@insa-lyon.fr

${ }^{3}$ Michel.frederic@cea.fr
} 


\section{INTRODUCTION}

The French Atomic and Alternatives Energies Commission, CEA, is working on the safety of the Sodium-cooled Fast Reactor (SFR). The monitoring of sodium pipes, between the reactor pool and the Sodium Gas Heat Exchanger (SGHE) is for example an important safety related issue. In such a context, liquid sodium leakage detection along pipes claims for new monitoring methodologies.

It is well-known, that due to the strong coupling between dense liquid and a pipe transporting it, a significant variation in the properties of the liquid flow modifies the dynamic behavior of the pipe. It results that the analysis of the pipe vibrations, measured from outside the pipe, may be an efficient way for detecting anomalies of the process sodium flow, such as the presence of leaks in a gas/sodium heat exchanger, possibly used in a SFR. The small leaks in the SGHE are characterized by the presence of gas bubbles in the sodium. The presence of bubbles can significantly altered the fluid flow properties (celerity, attenuation, linearity, etc.[1], [2]) and modify in a measurable way the vibratory behavior of the pipe. It is therefore interesting to analyze the vibratory behavior of the latter in order to quantify the bubbles. Many different techniques are proposed in the literature for bubble detection or measurement: optical methods [3], acoustic methods [4], electrical or electromagnetic methods [5], methods based on the emission of ionizing radiation [6]. However, due to the opacity of sodium and the complexity of the detection environment, the acoustic methods may be the best way to detect leaks on pipes.

For safety issues and seen the acoustic similarities between water at $20^{\circ} \mathrm{C}$ and sodium at $550^{\circ} \mathrm{C}$, for the present study, the fluid used inside the pipe is water (rather than sodium). One considers a straight pipe filled with a water/air mixture with a given void fraction. The pipe is coupled to a hydraulic circuit through two flanges.

On one hand, the presence of the latter have a significant influence on the vibroacoustic behavior of the pipe. Indeed, they may modify the vibration boundary conditions of the pipe and lead to wave reflexions at both flanges. The constructive interferences of these waves for some frequencies leads to a resonance phenomenon. On the other hand, the presence of bubble clouds in liquids is known to dramatically modify their acoustic properties. In fact, the radiation sound in the bubbly liquid is governed by the effective sound velocity of the two-phase mixture.

Therefore, we study the correlation between the resonance frequencies of the stiffened pipe and the void fraction in the fluid.

Let us consider a stiffened pipe section filled with a bubbly liquid and excited by a radial force point. The aim of this study is to analyze the vibratory response of the pipe. By exciting the stiffened pipe, one may observe a signal representing the vibratory level of the pipe displacements as a function of frequency. Thus, by identifying the modes and resonance frequencies of the pipe vibrations, one could estimate the characteristics of the liquid inside the stiffened pipe and then estimate the gas volume fraction. In parallel, a numerical model of the pipe coupled to two flanges has been developed to perform numerical experiments. This model is presented in section 2 while its exploitation to study the correlation between the resonance frequencies and the void fraction is presented in section 3 .

\section{THEORY FORMULATIONS OF A NUMERICAL MODEL OF A STIFFENED CYLIN- DRICAL SHELL FILLED WITH A BUBBLY LIQUID}

In the following section, an analytical model is presented to analyze the vibroacoustic behavior of a stiffened cylindrical shell filled with a bubbly liquid. The model resolution is based on the circumferential admittance approach (CAA) and a spectral decomposition of the vibratory and pressure fields. 
In fact, the presence of bubbles in the fluid must be taking account to predict the vibroacoutic behavior of a shell filled with a bubbly liquid.

The developed approach is therefore based on the combination of two models:

- A first model to relate the acoustic characteristics of the liquid to the void fraction. The gas volume fraction and sound velocity at frequencies much less than bubble resonances are related through a simple mathematical model known as Wood's equation [7]. This model is only applicable for working frequencies below the resonant frequencies of the bubbles present in the cloud. At frequencies below bubble resonance, the sound velocity is primarily dependent on the volume fraction of gas in the liquid.

- A second vibro-acoustic model to follow the progress of the resonant frequencies of the pipe according to the acoustic velocity of the liquid it contains. The model is based on a substructuring approach based on the circumferential admittance method coupled with a spectral approach of the shell filled with heavy fluid.

\subsection{Stiffened cylindrical shell model}

The system studied in this paper considers an infinite cylindrical shell filled with a heavy bubbly liquid coupled to two ring stiffeners as shown in Figure 1. A substructuring approach called the Circumferential Admittance Approach (CAA) is used for modeling the vibroacoustic behavior of a combined cylindrical shell with two circumferential stiffeners. This approach is based on the admittance principle for linear systems to calculate the harmonic response of subsystems coupled by point contacts. Thus, the shell and the stiffeners are considered as two subsystems independent one from the other.

As shown in Figure 1 a cylindrical coordinate system $(x, \mathrm{r}, \theta)$ is considered. The two ring stiffeners are located at the axial positions, $x_{1}$ and $x_{2}$.

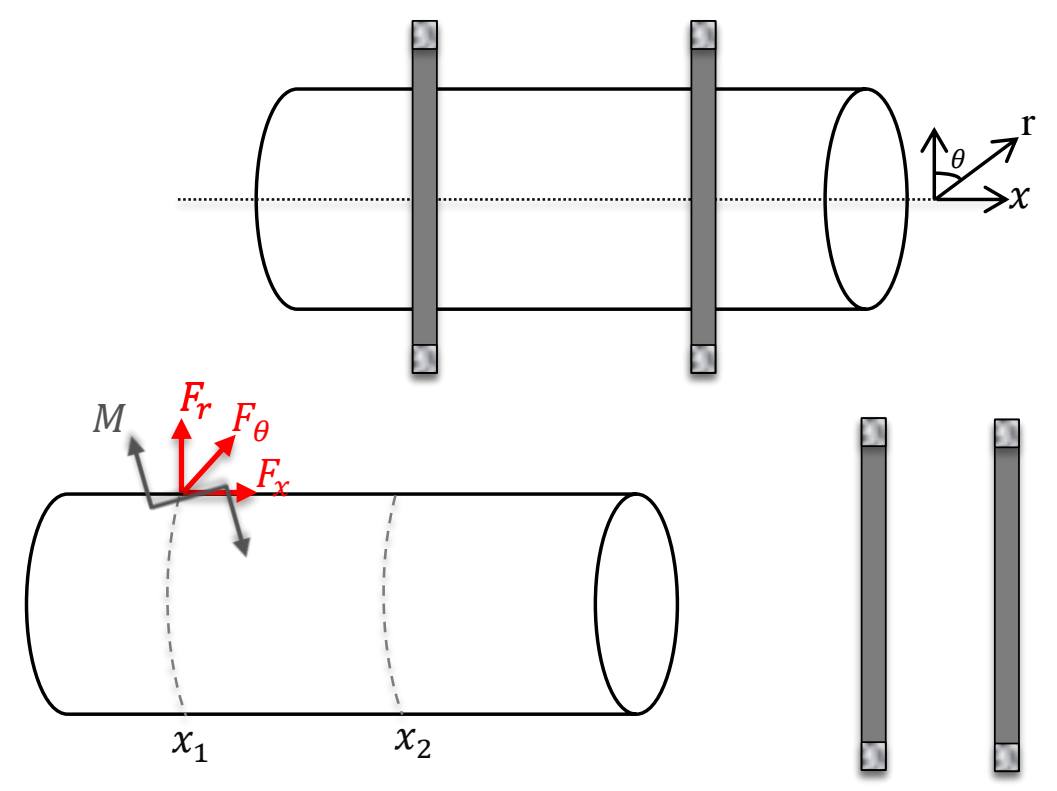

Figure 1: Illustration of cylindrical shell coupled to two ring stiffeners.

On one hand, the vibrations of the cylindrical shell and the stiffeners are respectively estimated by the Flügge model [8], [9] and a circular plate model (describing in-plane and out-of-plane vibrations) [10], while, the Helmholtz formulation is taken to model the fluid behavior. On the other hand, the 
junction between the shell and the stiffeners is assumed to be sufficiently rigid, which allows the two subsystems to have the same displacements and rotations at the points of contact. The resolution of the motion equations are performed using an accelerated spectral approach, which consists in modeling the problem in the high wavenumbers to improve convergence issues in the calculation of the admittances.

One defines the displacements and forces applied at the junction i $(i=1,2)$ between the two subsystems as follows:

- $U_{x, i}^{S}, U_{\theta, i}^{S}, U_{r, i}^{S}$ et $\varphi_{i}^{S}$ (respectively $U_{x, i}^{p}, U_{\theta, i}^{p}, U_{r, i}^{p}$ and $\left.\varphi_{i}^{p}\right)$ : the axial, tangential and radial displacements and $\varphi_{i}^{S}=\frac{\partial U_{r, i}^{S}}{\partial x}\left(\right.$ resp. $\left.\varphi_{i}^{p}=\frac{\partial U_{x, i}^{p}}{\partial r}\right)$ the angular rotation of the shell (respectively the ring-stiffener);

- $F_{x, i}^{S}, F_{\theta, i}^{S}, F_{r, i}^{S}$ et $M_{i}^{S}$ (respectively $F_{x, i}^{p}, F_{\theta, i}^{p}, F_{r, i}^{p}$ and $M_{i}^{p}$ ): the axial, tangential, radial forces, and the angular moment acting on the ring stiffener (respectively shell) by the shell (respectively ring stiffener).

The circumferential amplitudes of these quantities are defined by their Fourier series depending on circumferential orders $n$ :

$$
\begin{gathered}
\tilde{f}(n)=\frac{1}{2 \pi} \int_{0}^{2 \pi} f(\theta) e^{-j n \theta} d \theta, \quad \forall n \in \mathbb{Z} \\
f(\theta)=\sum_{n \in \mathbb{Z}} \tilde{f}(n) e^{j n \theta}
\end{gathered}
$$

Furthermore, for each subsystem $\alpha$, one defines the circumferential admittance between the junction $i$, and the junction $j$ as the ratio of the displacement at the junction $i$ to the applied force at the junction $j$ :

$$
\tilde{Y}_{\xi_{i} \zeta_{j}}^{\alpha}=\frac{\tilde{\xi}_{i}^{\alpha}}{\tilde{\zeta}_{j}^{\alpha}}, i \in\{1,2\}, j \in\{1,2\}
$$

where,

$$
\xi_{j}^{c} \in\left\{U_{x, j}^{\alpha}, U_{\theta, j}^{\alpha}, U_{r, j}^{\alpha}, \varphi_{j}^{\alpha}\right\} \text { et } \zeta_{j}^{\alpha} \in\left\{F_{x, j}^{\alpha}, F_{\theta j}^{\alpha}, F_{r, j}^{\alpha}, M_{j}^{\alpha}\right\}
$$

By making use of the superposition principle for linear passive systems and considering displacements continuity and equilibrium conditions at the junctions between the shell and the ring stiffeners, one obtains the following linear equation system:

$$
\left[\tilde{Y}^{\text {shell }}+\tilde{Y}^{\text {stiffeners }}\right]\left[\tilde{F}^{\text {coupling }}\right]=-\widetilde{\bar{W}}^{\text {shell }}
$$

where $\widetilde{\widetilde{W}}^{\text {shell }}$ represents the free displacement vector of the cylindrical shell when it is uncoupled from the ring stiffeners and only excited by an external radial point force. 


$$
\tilde{F}_{j}^{\text {coupling }}=\left[\begin{array}{c}
\tilde{F}_{x j}^{r} \\
\tilde{F}_{\theta j}^{r} \\
\tilde{F}_{r j}^{r} \\
\widetilde{M}_{j}^{r}
\end{array}\right] \quad \widetilde{W}^{\text {shell }}=\left[\begin{array}{c}
\widetilde{U}_{x j}^{s} \\
\widetilde{\bar{U}}_{\theta j}^{s} \\
\widetilde{\bar{U}}_{r j}^{s} \\
\widetilde{\bar{\varphi}}_{j}^{s}
\end{array}\right]
$$

and $\tilde{Y}^{\text {shell }}$ and $\tilde{Y}^{\text {stiffeners }}$ are the $4 \mathrm{x} 4$ circumferential admittance matrices of the shell and the ring stiffeners given by:

$$
\begin{aligned}
& \widetilde{Y}^{\text {shell }}=\left[\begin{array}{llll}
\widetilde{Y}_{U x_{i} F x_{j}}^{s} & \widetilde{Y}_{U x_{i} F \theta_{j}}^{s} & \widetilde{Y}_{U x_{i} F r_{j}}^{s} & \widetilde{Y}_{U x_{i} M_{j}}^{s} \\
\widetilde{Y}_{U \theta_{i} F x_{j}}^{s} & \widetilde{Y}_{U \theta_{i} F \theta_{j}}^{s} & \widetilde{Y}_{U \theta_{i} F r_{j}}^{s} & \widetilde{Y}_{U \theta_{i} M_{j}}^{s} \\
\widetilde{Y}_{U r_{i} F x_{j}}^{s} & \widetilde{Y}_{U r_{i} F \theta_{j}}^{s} & \widetilde{Y}_{U r_{i} F r_{j}}^{s} & \widetilde{Y}_{U r_{i} M_{j}}^{s} \\
\widetilde{Y}_{\varphi_{i} F x_{j}}^{s} & \widetilde{Y}_{\varphi_{i} F \theta_{j}}^{s} & \widetilde{Y}_{\varphi_{i} F r_{j}}^{s} & \widetilde{Y}_{\varphi_{i} M_{j}}^{s}
\end{array}\right] \\
& \widetilde{Y}^{\text {stiffeners }}=\left[\begin{array}{llll}
\widetilde{Y}_{U x_{i} F x_{j}}^{p} & \widetilde{Y}_{U x_{i} F \theta_{j}}^{p} & \widetilde{Y}_{U x_{i} F r_{j}}^{p} & \widetilde{Y}_{U x_{i} M_{j}}^{p} \\
\widetilde{Y}_{U \theta_{i} F x_{j}}^{p} & \widetilde{Y}_{U \theta_{i} F \theta_{j}}^{p} & \widetilde{Y}_{U \theta_{i} F r_{j}}^{p} & \widetilde{Y}_{U \theta_{i} M_{j}}^{p} \\
\widetilde{Y}_{U r_{i} F x_{j}}^{p} & \widetilde{Y}_{U r_{i} F \theta_{j}}^{p} & \widetilde{Y}_{U r_{i} F r_{j}}^{p} & \widetilde{Y}_{U r_{i} M_{j}}^{p} \\
\widetilde{Y}_{\varphi_{i} F x_{j}}^{p} & \widetilde{Y}_{\varphi_{i} F \theta_{j}}^{p} & \widetilde{Y}_{\varphi_{i} F r_{j}}^{p} & \widetilde{Y}_{\varphi_{i} M_{j}}^{p}
\end{array}\right]
\end{aligned}
$$

By inverting the system given in the Eq.5, we deduce the coupling forces $\tilde{F}_{j}^{\text {coupling }}$ exerted by the two ring stiffeners on the shell filled with fluid. These forces and moments are finally reinjected in the Flügge spectral model and a $2 D$ inverse Fourier transform is used in order to estimate the vibration field value of the shell when coupled to the ring stiffeners. (see for full details [11]).

\subsection{The Wood's model}

The bubble behaves acoustically like a pulsating sphere and radiates sound uniformly in all directions. The bubble's resonance frequency has first been obtained by Minnaert in its most simple form [12]:

$$
f_{M}=\frac{1}{2 \pi R} \sqrt{\frac{3 \gamma P_{0}}{\rho_{l}}}
$$

where $R$, is the radius of the bubble, $P_{0}$, is the external pressure in the liquid and $\gamma$ and $\rho_{l}$ are the polytropic exponent of the gas in the bubble, and the density of the liquid respectively. For an air bubble $(\gamma=1.4)$ in water $\left(\rho_{l}=1000 \mathrm{Kg} \cdot \mathrm{m}^{-3}\right)$ under normal conditions $\left(P_{0}=100 \mathrm{kPa}\right)$ the relation $f_{M} \cdot R \cong 3.26 \mathrm{kHz} \cdot \mathrm{mm}$ holds.

Wood was the pioneer to demonstrate the fact that the sound velocity is a function of volume fraction of the gas phase [7]. The Wood's model relates the sound velocity inside the cloud to the volume fraction of gas bubbles in the mixture. This sound velocity is strongly dependent upon the volume fraction of the gas, since the collective oscillation frequency is well below the resonance frequency of largest single bubble. The total volume fraction of the gas is known as the void fraction and, is given by: 


$$
\tau=\frac{V_{g}}{V_{m}}
$$

where $V_{g}$ and $V_{l}$ are the volume of the gas phase and the liquid phase respectively and $V_{m}$ is the total volume of the mixture, given by $V_{m}=V_{g}+V_{l}$. The effective mixture density and compressibility are:

$$
\left\{\begin{array}{l}
\rho_{m}=(1-\tau) \rho_{l}+\tau \rho_{g} \\
\chi_{m}=(1-\tau) \chi_{l}+\tau \chi_{g}
\end{array}\right.
$$

where $\rho_{l}$ is the density of the liquid and $\rho_{g}$ is the density of the gas. While $\chi_{l}$ is the compressibility of the liquid and $\chi_{g}$ is the compressibility due to the gas in the bubbles.

The sound velocity in an acoustic medium $i$ is defined by:

$$
\mathrm{C}_{i}=\frac{1}{\sqrt{\rho_{i} \chi_{i}}}
$$

Thanks to the definition of equivalent density and compressibility of the mixture by Eq.10, the phase velocity in a gas liquid mixture also known as Wood's velocity, is given by [7], [13] :

$$
\frac{1}{\mathrm{C}_{m}^{2}}=\frac{\tau^{2} \gamma}{\mathrm{C}_{g}^{2}}+\frac{(1-\tau)^{2}}{\mathrm{C}_{l}^{2}}+\tau(1-\tau)\left(\frac{\rho_{l}}{\mathrm{P}_{0}}+\rho_{g} \chi_{l}\right)
$$

where $\mathrm{P}_{0}$ and $\gamma$ are the pressure and adiabatic index of gas respectively. This equation is derived on the assumption that each individual bubble pulsates adiabatically, whereas the air/bubble mixture behaves undergoing an isothermal process.

The Figure 2 shows the plot of the Wood's model for a liquid water/air mixture under 1 bar at $20 \mathrm{C}^{\circ}$. For a void fraction as low as $10^{-6}$ a $1 \%$ decrease of the velocity is achieved. Whereas, for a $5.10^{-6}$ void fraction, this decrease reaches $5 \%$. We are therefore dealing with a mixture whose acoustical properties are very different from those of its constituents. This phenomenon could be easily verified on a daily basis thanks to the hot chocolate effect [14].

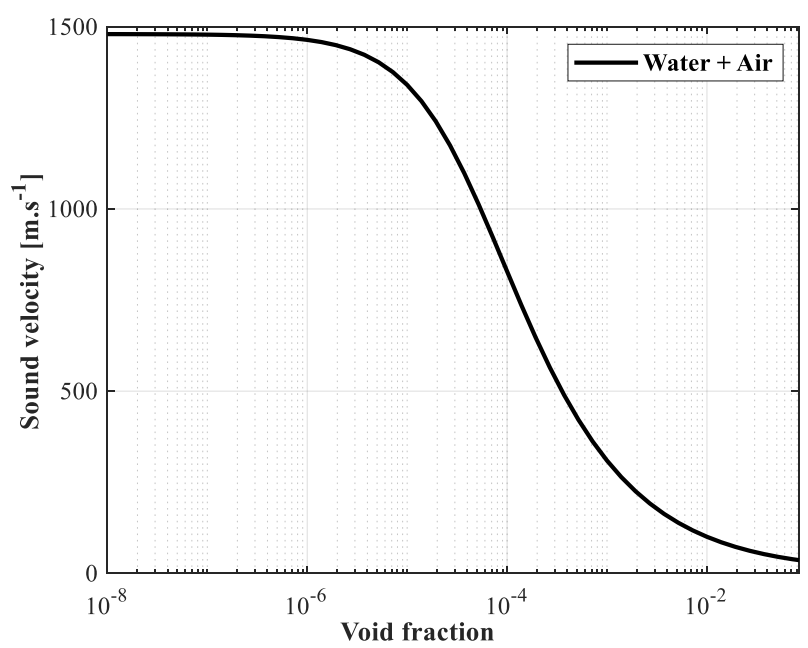

Figure 2 : The velocity in a liquid water/air mixture according to the Wood's model (1 bar at $20 \mathrm{C}^{\circ}$ ). 
We see on Figure 2, that if the sound velocity is known, the void fraction can be deduced on an important logarithmic range.

\section{NUMERICAL STUDY ON A STIFFENED CYLINDRICAL SHELL FILLED WITH A BUBBLY FLUID}

In this section, some numerical results of the present approach are presented for the mock-up considered for the laboratory experiment. It consists of a cylindrical pipe filled with a water/air mixture and coupled to two ring flanges located at $x_{1}$ and $x_{2}$. The pipe is characterized by an inner radius $R_{S}$ and a thickness $h_{s}$, while the ring flanges are characterized by a thickness $h_{p}$ and a height $l_{p}$. The pipe and the flanges are made of the same material, stainless steel, with Young's modulus E, Poisson's ratio $v$, and density $\rho$.

Tableau 1: Material characteristics and mock-up dimensions.

\begin{tabular}{ll}
\hline Stiffeners & Cylindrical shell \\
\hline$x_{1}=0 \mathrm{~m}$ & \\
$x_{2}=0,79 \mathrm{~m}$ & $h_{S}=0,004 \mathrm{~m}$ \\
$h_{p}=0,010 \mathrm{~m}$ & $R_{S}=0,06 \mathrm{~m}$ \\
$l_{p}=0,100 \mathrm{~m}$ & \\
\hline Young modulus & $E=2,110^{11} \mathrm{~Pa}$ \\
Structural damping coefficient & $\eta=0,01$ \\
Poisson coefficient & $v=0,3$ \\
Density & $\rho=7800 \mathrm{Kg} / \mathrm{m}^{3}$ \\
\hline
\end{tabular}

\subsection{Numerical validation for an invacuo shell coupled to the ring stiffeners}

A Finite elements model (FEM) was developed to check the validity of the analytical model that we have developed. Simulations was performed considered an invacuo pipe in order to consider only structural finite elements. Moreover, a pipe of finite length is considered in the finite element simulations whereas it is of infinite extend in the analytical model. A comparison of the results between the present approach and the finite element simulations is shown in Figure 3. It can be observed that the results of the present method are in good agreement with the FEM results. This allows us to validate the coupling the cylindrical shell and the ring stiffeners by the circumferential admittance approach. 


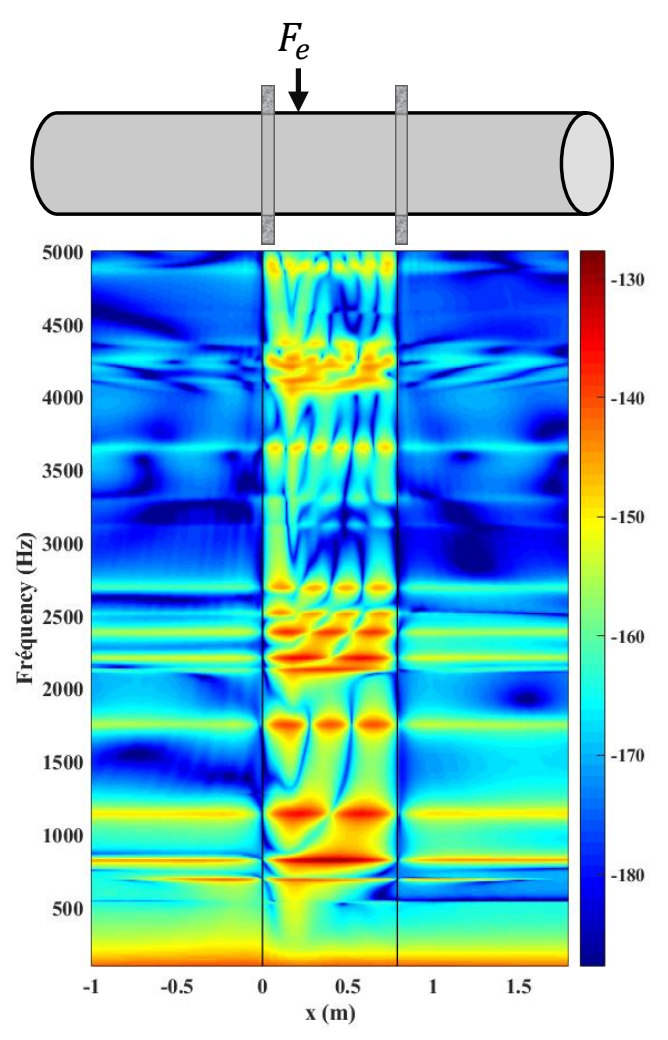

(a)

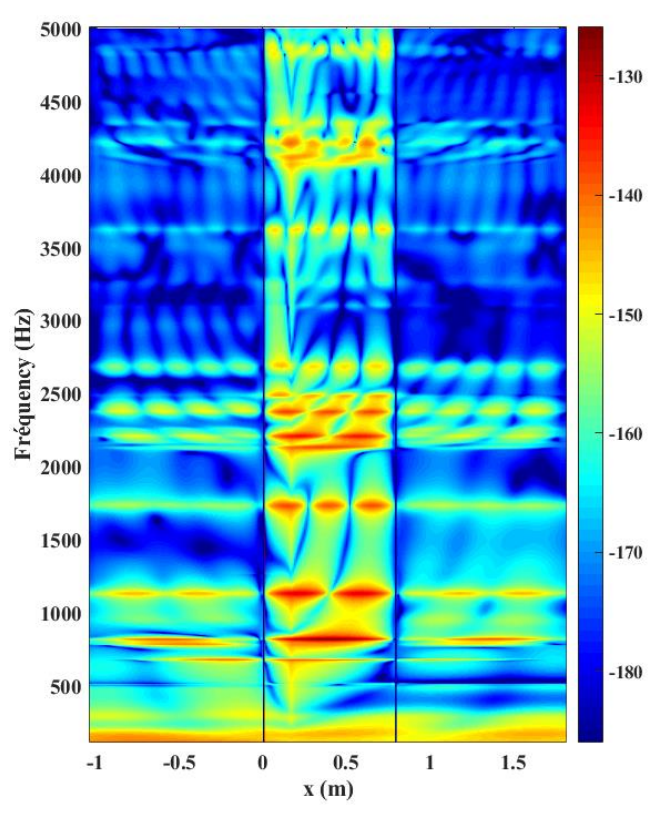

(b)

Figure 3 : Radial displacement level (ref.dB, $1 \mathrm{~m}$ ) in function of the axial position and the frequency of a shell invacuo coupled to two stiffeners and excited by a radial force point $F_{e}$ at $x_{e}=0.17 \mathrm{~m}$ and $\theta=0$. (a): Analytical model; (b): Finite Element Model.

\subsection{Numerical study of the correlations between the void fraction and the vibration re- sponses}

For this study, we suppose that the water is filled with air bubbles distributed homogeneously throughout the pipe. The density and speed of sound for water are $\rho_{l}=100 \mathrm{Kg} \cdot \mathrm{m}^{-3}$ and $C_{l}=$ $1460 \mathrm{~m} . \mathrm{s}^{-1}$, while, the density and speed of sound for gas are $\rho_{g}=1.3 \mathrm{Kg} \cdot \mathrm{m}^{-3}$ and $C_{g}=$ $340 \mathrm{~m} . \mathrm{s}^{-1}$ and the damping loss factors have been set to $0.1 \%$ for both fluids.

The celerity of the vibroaacoustic waves of the order $n=0$, representing breathing motion, is highly sensitive to the void fraction. However, as these waves propagate mainly in the fluid, they are not highly sensitive to the presence of stiffeners. In fact, resonant peaks can not be observed on the vibratory spectrum. Besides, their amplitudes are very low (these findings results are not presented in this paper).

In addition to the present measurement technique, other measurement techniques could be judicious to take advantage of the high sensitivity of the celerity to the void fraction for these orders. For example, using piezoelectric wires PVDF may offer an alternative to estimate the sound celerity [15].

For the order $n=1$, the vibratory spectrum of the shell shows resonant peaks that could be exploited to estimate the void fraction. However, their vibratory levels are low compared to the orders $n=2$ and $n=3$ which means the ovalisation and triangularisation waves dominate. We will focus on these orders. 
In this sense, one plots in Figure 4 the level of the radial displacement of the fluid filled shell for the orders $n=2$ and $n=3$. The two orders show a vibratory behavior similar to the one of a plate vibrating in bending. Indeed, the effect of the curvature of the shell becomes less important, thus leading to displacements of higher amplitude.

To their sensitivity to void fractions, the plots of the maximum radial displacement for orders $n=2$ and $n=3$ show the presence of well-marked resonance peaks with higher amplitude. In Figure 4, for orders $n=2$ and $n=3$, only a frequency shift of $1 \mathrm{~Hz}$ (up to $5000 \mathrm{~Hz}$ ) is observed between fluid without bubble and a bubbly fluid with $\tau=10^{-5}$. While one notices for $\tau=5.10^{-4}$ and $\tau=10^{-3}$ a significant frequency shift of the shell resonances, of more than $70 \mathrm{~Hz}$. Below $\tau=10^{-5}$, the sensibility of orders is somewhat less than at higher void fractions. These numerical results are encouraging, as this frequency shift could be easily experimentally measured for the void fractions of interest.

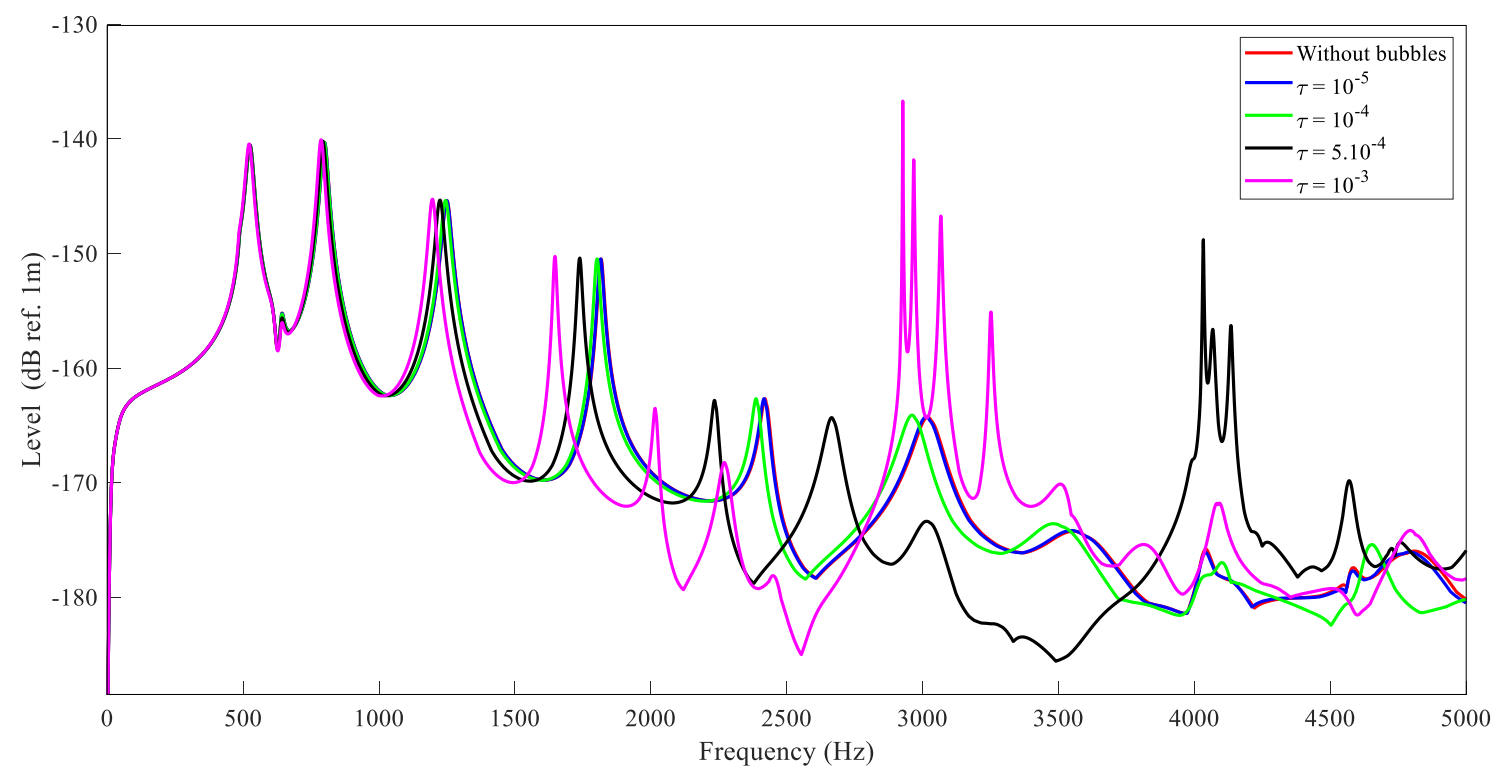

(a)

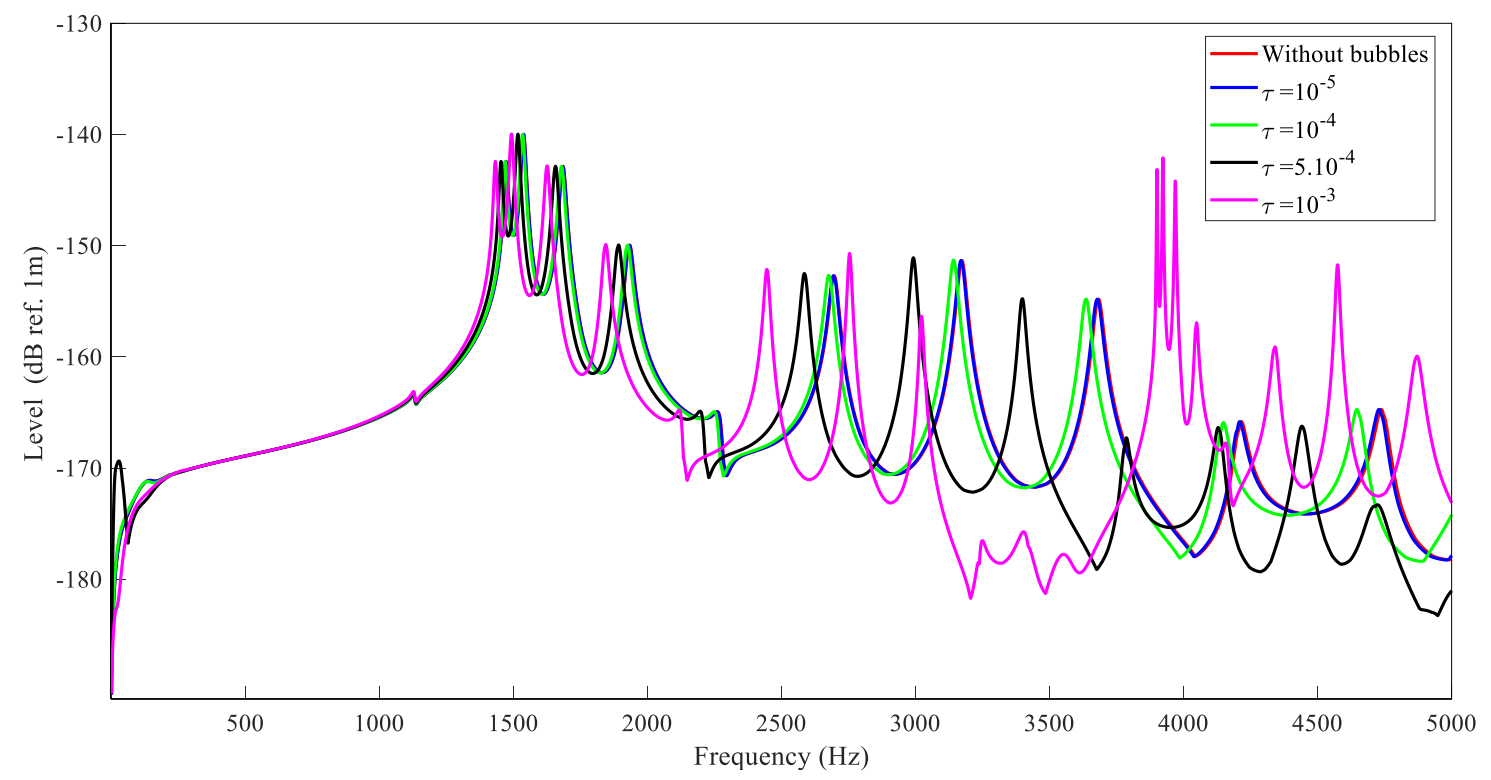

(b)

Figure 4: Level (dB, ref. $\left.1 \mathrm{~m}^{3}\right)$ of the radial displacement of the fluid filled shell under a radial force excitation point $F_{e}$ at $x_{e}=0.17 \mathrm{~m}$ and $\theta=0$, as a function of frequency. (a): order $n=2$, (b): order $n=3$. 


\section{CONCLUSIONS}

Some numerical results obtained from the developed analytical model are presented and discussed. The analytical model consists of a vibroacoustic model to predict the vibration responses of a cylindrical shell with infinite lateral extensions, filled with a bubbly liquid and coupled to two axisymmetric circumferential stiffeners (i.e. flanges). This model has been numerically validated for a cylindrical shell invacuo coupled to two axisymmetric stiffeners in a frequency range of $[0-4200] \mathrm{Hz}$. Indeed, the same results would be found by a FEM simulation. According to this validation, the model was used to study the numerical correlations between the void fraction and the vibratory characteristics of the stiffened pipe. The study allowed to analyze the vibratory behavior of the pipe for different void fractions and thus to identify the most sensitive vibration modes when the fluid characteristics changes and which order may be easily measured experimentally.

The vibrational levels related to the circumferential order $n=2$ and $n=3$ are higher than the ones related to the lower orders $(n=0$ and $n=1)$.

In addition, the $n=2$ and $n=3$ components of the vibratory field present a high sensitivity to the presence of gas in the fluid. The frequency shifts related to these orders due to a change of gas fraction would be easily measured experimentally. A comparison of numerical results with experimental results would be required.

In order to experimentally validate the developed analytical model, a hydraulic facility was set up. The aim of this loop is to further experiment under conditions closer to real industrial conditions: large and fully bubbly volume, variable void rates. The microbubble generation is based on the cavitation of air-oversaturated water. As the estimation of the void fraction is difficult to predict, we use an optical device to get reference measurements of the bubble clouds. A test campaign is underway.

\section{ACKNOWLEDGEMENTS}

This work is a part of a $\mathrm{PhD}$ thesis entitled "Detection of bubble presence in a dense liquid using vibration measurements". This research is performed in collaboration with CEA (Commissariat à l'Energie Atomique et aux Energies Alternatives) and Laboratory of Vibrations and Acoustic in INSA Lyon.

\section{REFERENCES}

[1] H. R. A. Mallock, « The damping of sound by frothy liquids », Proc. R. Soc. Lond. Ser. Contain. Pap. Math. Phys. Character, vol. 84, no 572, p. 391-395, déc. 1910, doi: 10.1098/rspa.1910.0085.

[2] K. W. Commander and A. Prosperetti, «Linear pressure waves in bubbly liquids: Comparison between theory and experiments », J. Acoust. Soc. Am., vol. 85, n 2, p. 732-746, févr. 1989, doi: 10.1121/1.397599.

[3] J. Garnier, E. Manon, and G. Cubizolles, «Local measurements on flow boiling of refrigerant 12 in a vertical tube », Multiph. Sci. Technol., vol. 13, no $1 \&$ 2, 2001, doi: 10.1615/MultScienTechn.v13.i1-2.10.

[4] L. D'Hondt, C. Payan, S. Mensah, and M. Cavaro, « Acoustic characterization of microbubble clouds by attenuation and celerity spectroscopy », J. Acoust. Soc. Am., vol. 141, n ${ }^{\circ}$ 5, p. 3506-3506, mai 2017, doi: 10.1121/1.4987347.

[5] W. Xu, K.-J. Xu, J.-P. Wu, X.-L. Yu, and X.-X. Yan, «Peak-to-peak standard deviation based bubble detection method in sodium flow with electromagnetic vortex flowmeter », Rev. Sci. Instrum., vol. 90, $\mathrm{n}^{\mathrm{o}}$ 6, p. 065105, 2019, doi: 10.1063/1.5089690.

[6] T. Aoyama, C. Ito, K. Okazaki, H. Harano, K. Watanabe, and T. Iguchi, « Development of Sodium Leak Detection Technology Using Laser Resonance Ionization Mass Spectrometry », J. 
Nucl. Sci. Technol., vol. 45, $\mathrm{n}^{\mathrm{o}}$ sup6, p. 43-50, sept. 2008, doi: 10.1080/00223131.2008.10875975.

[7] Wood A. B., A Textbook Of Sound. 1946.

[8] W. Flügge, Statik und Dynamik der Schalen. Springer-Verlag, 2013.

[9] «Expressions for direct evaluation of wave number in cylindrical shell vibration studies using the Flügge equations of motion: The Journal of the Acoustical Society of America: Vol 119, No $6 »$.

[10] Y. Tso and C. Hansen, « Wave-Propagation Through Cylinder Plate Junctions », J. Sound Vib., vol. 186, no 3, p. 447-461, sept. 1995, doi: 10.1006/jsvi.1995.0460.

[11] L. Maxit and J.-M. Ginoux, "Prediction of the vibro-acoustic behavior of a submerged shell non periodically stiffened by internal frames », J. Acoust. Soc. Am., vol. 128, n 1, p. 137-151, juill. 2010, doi: 10.1121/1.3436526.

[12] M. M. Sc.D, «XVI. On musical air-bubbles and the sounds of running water », Lond. Edinb. Dublin Philos. Mag. J. Sci., vol. 16, $\mathrm{n}^{\mathrm{o}}$ 104, p. 235-248, 1933, doi: 10.1080/14786443309462277.

[13] P. S. Wilson and R. A. Roy, «An audible demonstration of the speed of sound in bubbly liquids », Am. J. Phys., vol. 76, no 10, p. 975-981, oct. 2008, doi: 10.1119/1.2907773.

[14] F. S. Crawford, « The hot chocolate effect », Am. J. Phys., vol. 50, n 5, p. 398-404, 1982, doi: 10.1119/1.13080.

[15] G. Pavić and F. Chevillotte, «Cavitation monitoring in piping systems », Proc. ISMA Leven Belg., 2012. 